\title{
Light Localization in Disordered Metamaterials
}

\author{
Salvatore Savo", Nikitas Papasimakis and Nikolay Zheludev *** \\ Optoelectronics Research Centre and Centre for Photonic Metamaterials, University of Southampton, \\ Highfield, Southampton SO17 1BJ, United Kingdom \\ *ss2d09@orc.soton.ac.uk; ${ }^{* *}$ niz@orc.soton.ac.uk
}

Localization due to multiple scattering of photons in disordered electromagnetic media has triggered a recent paradigm shift in photonics, where disorder is no longer considered an unwanted disturbance on perfect periodicity, but is being used to achieve enhancement of luminescence, nonlinear optical interactions, Raman scattering and occupies a central position in random lasers. Here for the first time we extend the study of light localization to planar metamaterials. We study a metamaterial system consisting of asymmetrically-split ring (ASR) resonators that support both collective and individual modes. In such a metamaterial, a high-quality subradiant (trapped) modes can be excited that corresponds to a collective excitation of magnetic dipoles normal to the array plane and hence uncoupled to the magnetic field of the incident wave. This leads to a nearly-closed system, very weakly coupled to free-space, ideal for the study of localization effects.

We observed localization of microwave radiation in disordered ASR arrays under plane wave illumination by mapping the intensity profile of the near-field with a spectroscopic microwave scanning near-field instrument. Positional disorder was introduced by displacing the centre of each unit cell according to a random uniform distribution. Characteristic near-field intensity maps of the electric field component normal to the plane of the arrays at the resonance frequency of the subradiant mode $(5.8 \mathrm{GHz})$ are shown in Fig. 1. Whereas a regular sample presents a periodic pattern (Fig. 1a), upon introducing disorder a speckle pattern develops (see Fig. 1b) with certain rings appearing brighter (strong excitation) or darker (weak excitation).
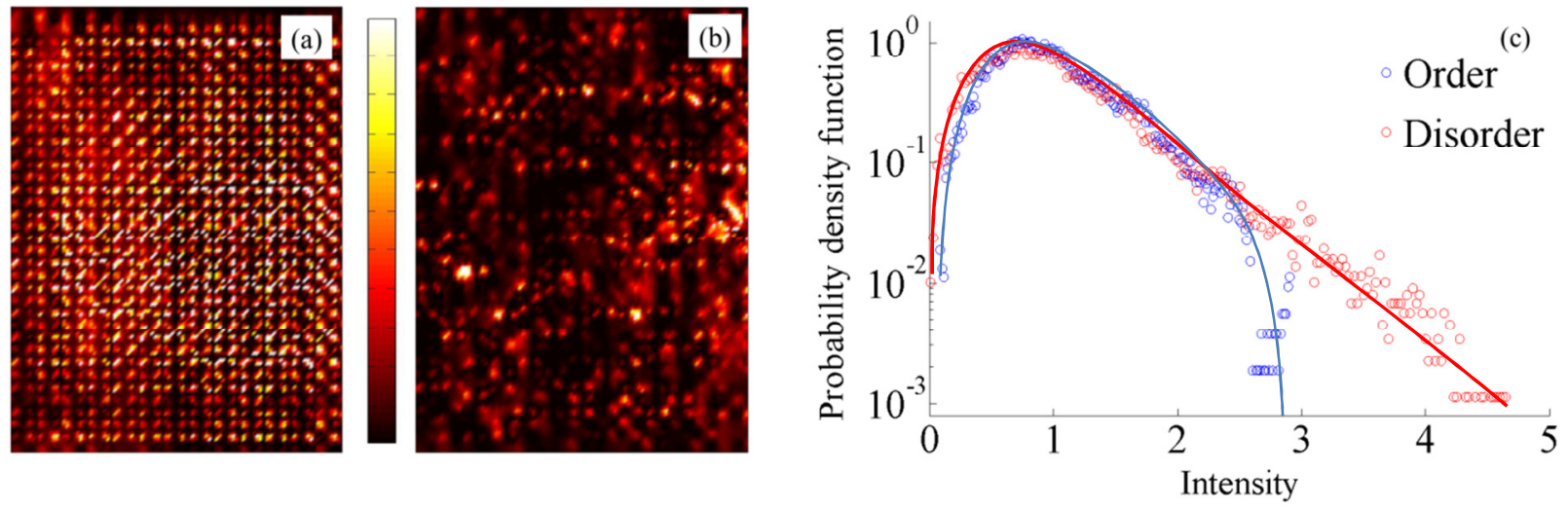

Fig.1. Characteristic near field maps for regular (a) and weakly (b) disordered metamaterials measured at 5.80GHz. (c) Probability density function of the normalized average values of the unit cells electric field intensities collected at the trapped mode resonance band. Red dots correspond to the disordered arrays, whereas blue dots refer to the regular arrays. Each point represents the average value of the intensity of the electric field $I$ over a single unit cell. Each unit cell is mapped with approximately 36 pixels.

The statistics of the near field intensity are studied by the probability density function of the electric nearfield intensity over a narrow band centred at the trapped mode resonance. In the case of the regular array the distribution decays rapidly with increasing intensity (blue curve in Fig. 1c), while disorder modifies dramatically the situation leading to a skewed distribution with a long tail at high intensities which is a typical signature of localization.

In conclusion, we study experimentally localization in planar metamaterial systems that support subradiant collective modes of excitation. The role of such collective modes and possible applications will be discussed. 